Open Access

\title{
Production of phenylacetyl-homoserine lactone analogs by artificial biosynthetic pathway in Escherichia coli
}

\author{
Sun-Young Kang 1,2, Jae Kyoung Lee ${ }^{1,2}$, Jae-Hyuk Jang ${ }^{1}$, Bang Yeon Hwang ${ }^{2}$ and Young-Soo Hong ${ }^{\text {** }}$
}

\begin{abstract}
Background: Quorum sensing (QS) networks are more commonly known as acyl homoserine lactone (HSL) networks. Recently, $p$-coumaroyl-HSL has been found in a photosynthetic bacterium. $p$-coumaroyl-HSL is derived from a lignin monomer, $p$-coumaric acid, rather than a fatty acyl group. The $p$-coumaroyl-HSL may serve an ecological role in diverse QS pathways between $p$-coumaroyl-HSL producing bacteria and specific plants. Interference with QS has been regarded as a novel way to control bacterial infections. Heterologous production of the QS molecule, p-coumaroyl-HSL, could provide a sustainable and controlled means for its large-scale production, in contrast to the restricted feedback regulation and extremely low productivity of natural producers.

Results: We developed an artificial biosynthetic process for phenylacetyl-homoserine lactone analogs, including cinnamoyl-HSL, p-coumaroyl-HSL, caffeoyl-HSL, and feruloyl-HSL, using a bioconversion method via E. coli (CB1) in the co-expression of the codon-optimized Luxl-type synthase (Rpal) and p-coumaroyl-CoA ligase (4CL2nt). In addition to this, we show the de novo production of $p$-coumaroyl-HSL in heterologous host E. coli (DN1) and tyrosine overproducing E. coli (DN2), containing the rpal gene in addition to $p$-coumaroyl-CoA biosynthetic genes. The yields for p-coumaroyl-HSL reached $93.4 \pm 0.6$ and $142.5 \pm 1.0 \mathrm{mg} / \mathrm{L}$ in the S-adenosyl-L-methionine and L-methionine feeding culture in the DN2 strain, respectively.
\end{abstract}

Conclusions: This is the first report of a de novo biosynthesis in a heterologous host yielding a QS molecule, $p$-coumaroyl-HSL from a glucose medium using a single vector system combining $p$-coumaroyl-CoA biosynthetic genes and the Luxl-type synthase gene.

Keywords: Homoserine lactone (HSL), Phenylacetyl-HSL, p-coumaroyl-HSL, Artificial biosynthesis

\section{Background}

Bacteria use small molecules and peptides to assess their local population densities in a process termed quorum sensing (QS) $[1,2]$. Quorum sensing is a regulatory system used by bacteria for controlling gene expression in response to increasing cell density $[3,4]$. When bacteria reach a sufficiently high population density, they will alter gene expression so as to carry out a range of processes that require the cooperation of a large number of cells

\footnotetext{
*Correspondence: hongsoo@kribb.re.kr

${ }^{1}$ Chemical Biology Research Center, Korea Research Institute

of Bioscience and Biotechnology, 30 Yeongudanji-ro, Ochang-eup,

Chungbuk 363-883, Republic of Korea

Full list of author information is available at the end of the article
}

[5]. These regulatory processes are remarkable in their diversity, ranging from virulence factor and antibiotic production to biofilm formation, root nodulation, and bioluminescence, and have direct and often devastating impacts on the bacterial host [6]. Many bacteria use acyl-HSL synthases to generate fatty acyl-HSL quorumsensing signals, which function with signal receptors to control the expression of specific genes. The fatty acyl group is derived from fatty acid biosynthesis and provides signal specificity, but the variety of signals is limited [2].

Meanwhile, the photosynthetic bacterium Rhodopseudomonas palustris produces a natural phenylacetyl-HSL, p-coumaroyl-HSL, using a LuxI-type synthase (RpaI, also 
known as $p$-coumaryl-homoserine lactone synthase, EC 2.3.1.229) with a $p$-coumaric acid rather than fatty acids $[7,8]$. Schaefer et al. speculated that there is an intimate relationship between $p$-coumaroyl-HSL producing bacteria and specific plants through $p$-coumaroyl-HSL signaling [7], because the rpaI gene expression is activated specifically by growth of $R$. palustris on $p$-coumaric acid [9], a major aromatic monomer of lignin, which comprise over $30 \%$ of all plant dry material. It is clear that several photosynthetic bacteria and nitrogen-fixing bacteria respond in complex ways to the presence of exogenous lignin monomers and this may drive intercellular signaling for a metabolites' production $[7,10]$. These metabolites may comprise antibiotics and auxins that suppress the growth of potentially parasitic bacteria and promote algal growth, respectively [11-13]. It is a distinctly possible scenario that phenylacetyl-HSL could serve an ecological role in these diverse QS pathways in natural environments.

Recently there have been intensive efforts by several groups to find small molecules that can interrupt the QS communication among bacteria [12, 14-20]. It is believed that disrupting bacterial communication and hence virulence factor production would not put substantial evolutionary pressure on bacteria to develop resistance. To address these challenges, non-native synthetic phenyl or phenol HSL analogs have shown significant activity against TraR, a QS receptor, in Agrobacterium tumefaciens and were 1-2 orders of magnitude more active than the previously reported LuxR-type protein antagonists examined as controls. Impressively, bromophenyl-HSL displayed $50 \%$ inhibition at an equimolar concentration of natural fatty acyl-HSL, N-3-oxooctanoyl-HSL [15-17, 20]. Interestingly, a close relative of $p$-coumaroyl-HSL, cinnamoyl-HSL, was shown to have limited activity against reporter strains for traditional HSLs [16]. These phenylacetyl-HSL compounds highlight the potential for autoinducer libraries with substantial structural diversity, to serve as probes or modulators for QS circuits mediated by 'nonstandard' signals [21].

Homoserine lactone biosynthesis typically involves a series of reactions that use S-adenosyl methionine (SAM) as the amino donor to generate the HSL ring moiety, and fatty-acyl carrier protein (ACP) or -acyl coenzyme A (CoA) as the precursor for the $\mathrm{N}$-acyl side chain of the HSL molecules $[11,22]$. When grown in the presence of $p$-coumaric acid, a few bacteria, $R$. palustris, Bradyrhizobium sp. BTAil and Silicibacter pomeroyi DSS-3, produced relatively small amounts $(10 \mu \mathrm{M})$ of p-coumaroyl-HSL [7]. In this study, we tested the substrate specificity of RpaI towards phenolic acids CoA e.g., cinnamoyl-, $p$-coumaroyl-, caffeoyl-, and feruloyl-CoA. In addition to this, we show the de novo production of
$p$-coumaroyl-HSL in heterologous host E. coli containing an artificial biosynthetic pathway that contained the rpaI gene in addition to $p$-coumaroyl-CoA biosynthetic genes. The production of $p$-coumaroyl-HSL was about three fold higher in the engineered tyrosine overproducing $E$. coli strain compared to that of the wild type E. coli. Finally, the yields for $p$-coumaroyl-HSL were $93.4 \pm 0.6$ and $142.5 \pm 1.0 \mathrm{mg} / \mathrm{L}$, respectively, by the tyrosine overproducing $E$. coli with SAM, or the L-methionine feeding strategy.

\section{Results and discussion}

In vitro enzymatic synthesis of phenolic acids to phenylacetyl-homoserine lactone analogs

RpaI, a LuxI-type synthase, was demonstrated to be a $p$-coumaroyl-HSL synthase [7]. On the basis of this precedent, we attempted enzymatic production using the purified His-tagged RpaI with various phenolic acids$\mathrm{CoA}$, in order to make novel phenylacetyl-HSL analogs (Fig. 1). In order to complete an initial survey of the substrate specificity of the RpaI enzyme, as a start the $p$-coumaric acid-CoA ligase (4CL2nt, EC 6.2.1.12) and four phenolic acids (cinnamic acid, $p$-coumaric acid, caffeic acid, and ferulic acid) were used in our in vitro enzyme reaction. The 4CL2nt from Nicotiana tabacum had already been identified with a broad substrate specificity for cinnamic acid, $p$-coumaric acid, caffeic acid, and ferulic acid [23]. The reaction of each phenolic acid with 4CL2nt enzyme in the presence of Rpal led to the formation of a new product, which was detected by HPLC (Fig. 2).

The putative phenylacetyl- HSL analogs appeared as a UV spectrum pattern similar to $p$-coumaroyl-HSL (broad peak of maximum absorbance at $308 \mathrm{~nm}$ and a minor peak at $227 \mathrm{~nm}$ ) in the HPLC analyses (Additional file 1: Figure S1). These compounds were further analyzed using liquid chromatography-mass spectrometry (LCMS) (Additional file 1: Figure S2). The peak at $m / z 248$ $[\mathrm{M}+\mathrm{H}]^{+}$, which corresponded to $p$-coumaroyl-HSL, was detected in significant amounts with $p$-coumaric acid as a precursor. It was expected that the $p$-coumaric acid converted to $p$-coumaroyl-HSL through lactonization reaction of the $p$-coumaroyl-CoA.

In addition, we found three putative HSL peaks in LC/MS analyses using cinnamic acid, caffeic acid, and ferulic acid as each starter substrate; each peak showed a molecular ion at $\mathrm{m} / \mathrm{z} 232, \mathrm{~m} / \mathrm{z} 264$, and $\mathrm{m} / \mathrm{z} 278$ $[\mathrm{M}+\mathrm{H}]^{+}$, respectively (Additional file 1: Figure S2). A full scan and $\mathrm{MS}^{2}$ mass spectral data for this putative lactone product showed a loss of $102 \mathrm{Da}$ (HSL moiety) from the parent ion, which is a distinguishing pattern of the phenylacetyl-HSL analogs. When the relative HPLC peak area was calculated based on a quantitative 


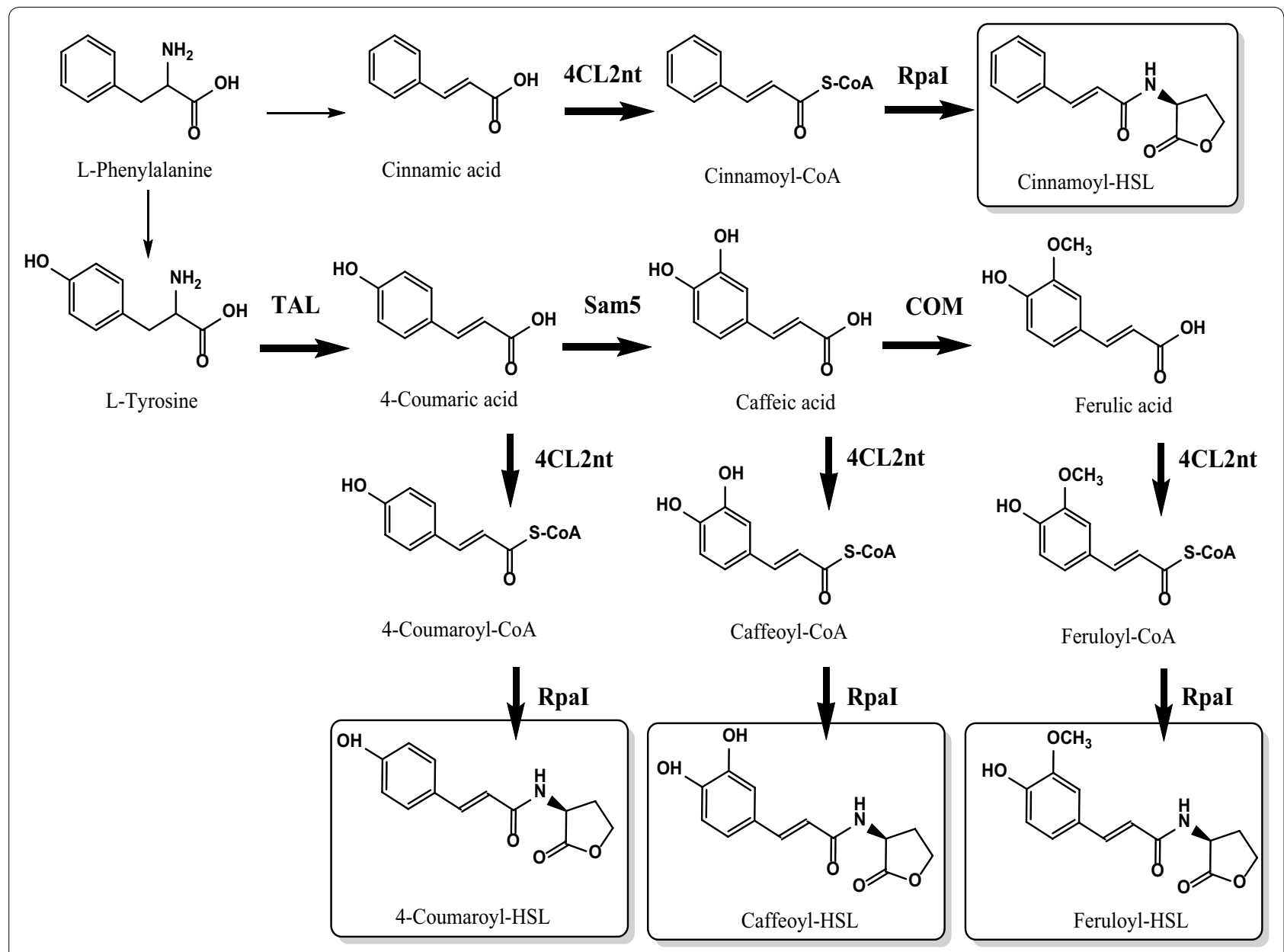

Fig. 1 Engineered biosynthetic pathways for the phenylacetyl-HSL analogs in E. coli. The acyl-HSL synthase Rpal from Rhodopseudomonas palustris catalyzes the conversion of phenolic acid coenzyme A to phenylacetyl-HSL. The phenolic acid substrate is achieved through the conversion of L-tyrosine to $p$-coumaric acid by a tyrosine ammonia lyase (TAL) from Saccharothrix espanaensis, hydroxylation of $p$-coumaric acid to caffeic acid by Sam5 from S. espanaensis, and O-methylation of caffeic acid to ferulic acid by O-methyltransferase (COM) from Arabidopsis thaliana. A 4-coumarateCoA ligase from Nicotiana tabacum (4CL2nt) generates the CoA-ester of the phenolic acid substrates, allowing lactone formation catalyzed by Rpal

comparison with the substrates and the products after reactions, roughly $34,47,72$, and $46 \%$ conversion ratios were shown for cinnamic acid, $p$-coumaric acid, caffeic acid, and ferulic acid, respectively (Fig. 3). Interestingly, the relative conversion ratio with caffeic acid is higher than the ratio of $p$-coumaric acid, which is the previously reported precursor of the RpaI enzyme [7]. In addition, the 4CL2nt had already confirmed that the relative activities toward caffeic acid were $25 \%$, compared to $p$-coumaric acid as substrates [23]. Taking these results together, we considered the possible explanation that the caffeoyl-CoA was a more suitable substrate for the RpaI enzyme. But, we found it difficult to analyze the RpaI enzyme kinetic data to obtain valid substrate specificity, because the caffeoyl-CoA compound was not commercially available.

\section{Bioconversion of phenolic acids} to phenylacetyl-homoserine lactone analogs in $E$. coli In order to more effectively obtain an amount of the novel phenylacetyl-HSL analogs, we constructed a bioconversion system for production of the novel phenylacetyl-HSL analogs utilizing the codon-optimized p-coumaroyl-HSL synthase gene (rpaI) addition with $p$-coumaroyl-CoA ligase gene $(4 c l 2 n t)$. The synthetic rpaI and $4 c l 2 n t$ genes were cloned into the expression vector pET-28a $(+)$ using previously described cloning methods $[24,25]$, which resulted in pET-4R (Table 1 ). The four phenolic acids were added to the cultured recombinant E. coli C41(DE3) strain (CB1) with the rpaI and 4cl2nt genes (pET-4R). The CB1 culture broth and bacterial cells were collected after $24 \mathrm{~h}$ culture and were then subjected to HPLC analyses (Fig. 4). Under the bioconversion 

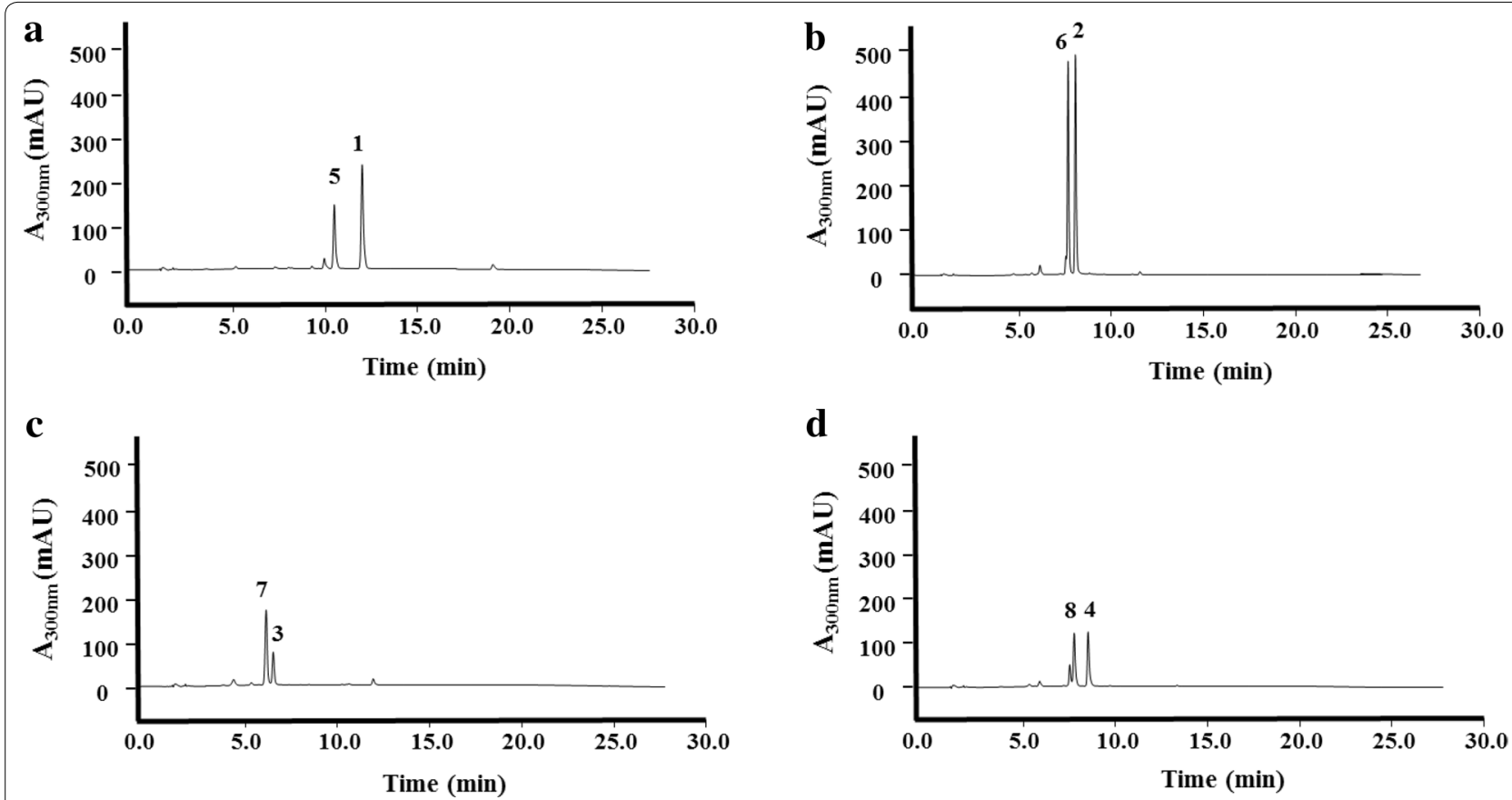

Fig. 2 HPLC analysis of the in vitro enzymatic reaction with p-coumaric acid CoA ligase (4CL2nt) and acyl-HSL synthase (Rpal). The detection wavelength was $300 \mathrm{~nm}$ for reactions with cinnamic acid (a), p-coumaric acid (b), caffeic acid (c) and ferulic acid (d). Peak 1, cinnamic acid; peak 2, p-coumaric acid; peak 3, caffeic acid; peak 4, ferulic acid; peak 5, cinnamoyl-HSL; peak 6, p-coumaroyl-HSL; peak 7, caffeoyl-HSL; peak 8, feruloyl-HSL

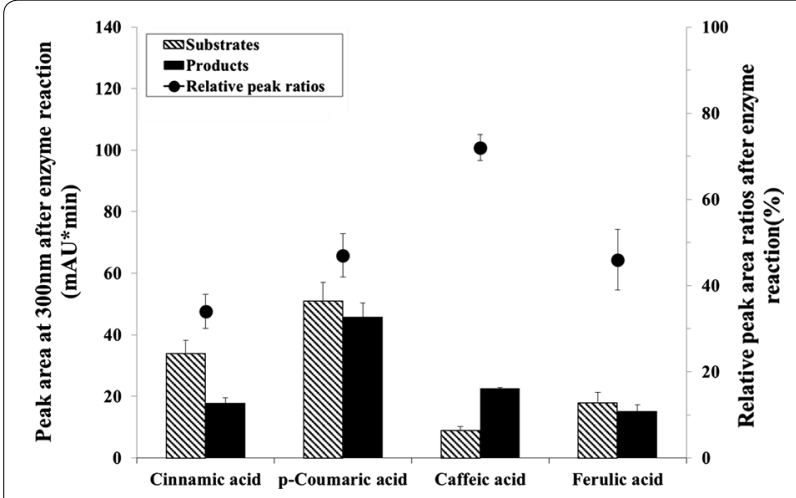

Fig. 3 Relative HPLC peak area ratio between each product and substrate after enzymatic reactions. The HPLC peak area of remaining substrates (lines) and acyl-HSL products (black) were analyzed after each enzymatic reaction with cinnamic acid, $p$-coumaric acid, caffeic acid or ferulic acid. The ratios (circles) were calculated by peak area of each product against each remaining substrate at the end of enzymatic reactions. The detection wavelength was $300 \mathrm{~nm}$ for reactions with each phenolic acid. Error bars reported at one standard deviation from triplicate experiments

condition employed in this study, cinnamic acid, $p$-coumaric acid, caffeic acid, and ferulic acid were each converted to phenylacetyl-HSL, respectively.

To obtain NMR-accessible amounts from the present bioconversion conditions, $20 \mathrm{mg}$ of each phenolic acid were added to a $2 \mathrm{~L}$ fermentation of CB1 strain. The structures of the purified phenylacetyl-HSL analogs were identified through spectral data interpretation and compared with the values reported in the literature [7]. The presence of HSL ring moieties were revealed by the ${ }^{1} \mathrm{H}$ NMR data $\left[\delta_{\mathrm{H}} 4.68-4.70(1 \mathrm{H}, \mathrm{H}-10), 4.24-4.39(2 \mathrm{H}\right.$, $\mathrm{H}-12)$, and 2.21-2.48 (2H, H-13)] for all compounds. Additionally, three exchangeable proton signals were observed in DMSO- $d_{6}$, at $\delta_{\mathrm{H}} 9.42(1 \mathrm{H}, 4-\mathrm{OH}), 9.16(1 \mathrm{H}$, $3-\mathrm{OH})$, and $8.52(1 \mathrm{H},-\mathrm{NH})$ which were assigned as two hydroxyl protons at the benzene ring and amide proton of caffeoyl-HSL. And also, feruloyl-HSL showed one methoxyl group at $\delta_{\mathrm{H}} 3.81(3 \mathrm{H}, 3-\mathrm{OCH})$ and one hydroxyl group at $\delta_{\mathrm{H}} 9.49(1 \mathrm{H}, 4-\mathrm{OH})$ on the benzene ring. In the ${ }^{1} \mathrm{H}$ NMR spectra, the large coupling constant $\left(J_{7,8}=15.7 \mathrm{~Hz}\right)$ implied the ${ }_{E}$-olefin relationship between C-7 and C-8 at the aryl side chain of each phenylacetylHSL compounds (Additional file 1: Tables S1, S2).

\section{Construction of de novo artificial biosynthetic pathways in $E$. coli to produce $p$-coumaroyl-HSL}

In addition, although the production of $p$-coumaroylHSL has been established by the above enzymatic reaction and bioconversion, we also describe a different approach for their de novo synthesis in $E$. coli by engineering an artificial biosynthetic pathway. This could be a useful approach for economic production by one-pot 
Table 1 Plasmids and strains used in this study

\begin{tabular}{|c|c|c|}
\hline Plasmid or strain & Relevant characteristics & References \\
\hline \multicolumn{3}{|l|}{ Plasmid } \\
\hline pET-28a $(+)$ & f1 ori, T7 promoter, Kan $^{R}$ & Novagen \\
\hline pET-opTAL & pET-28a $(+)$ carrying codon-optimized tyrosine ammonia lyase gene (tal) & Kang et al. [30] \\
\hline pET-4CL2nt & pET-28a $(+)$ carrying codon-optimized p-coumaroyl CoA ligase 4CL2 gene (4cl2nt) & This study \\
\hline pET-opRpal & pET-28a(+) carrying codon-optimized homoserine synthase gene (rpal) & This study \\
\hline pET-4R & pET-28a $(+)$ carrying codon-optimized 4cl2nt and rpal & This study \\
\hline pET-opT4R & pET-28a $(+)$ carrying codon-optimized tal, 4cl2nt and rpal & This study \\
\hline \multicolumn{3}{|l|}{ Strain } \\
\hline E. coli DH5a & Cloning host & Invitrogen \\
\hline E. coli C41(DE3) & Derivative strain of E. coli BL21(DE3) & Miroux and Walker [31] \\
\hline$\triangle \operatorname{COS} 1$ & E. coli C41(DE3); $\triangle t y r R:: t y r A^{\mathrm{fbr}}$, aro $\mathrm{G}^{\mathrm{fbr}}$; tyrosine overproducing strain & Kang et al. [27] \\
\hline CB1 & E. coli C41(DE3) harboring pET-4R & This study \\
\hline DN1 & E. coli C41(DE3) harboring pET-opT4R & This study \\
\hline DN2 & E. coli $\triangle \operatorname{COS} 1$ harboring pET-opT4R & This study \\
\hline
\end{tabular}
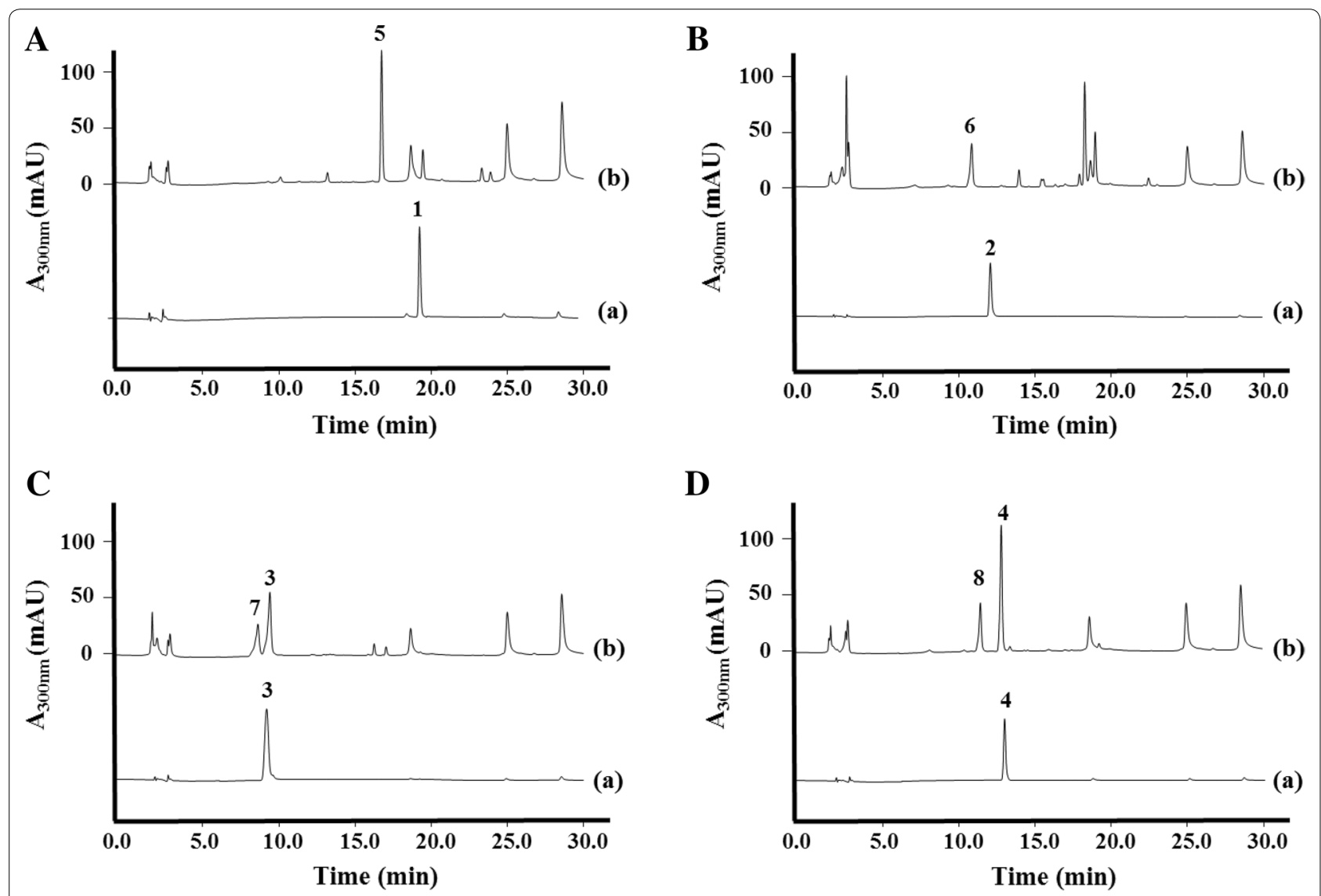

Fig. 4 Bioconversion experiments with each phenolic acid. A HPLC profile of the standard cinnamic acid (a) and cinnamic acid supplemented $E$. coli C41 (DE3) harboring pET-4R (CB1) (b); B standard p-coumaric acid (a) and p-coumaric acid supplemented E. coli harboring pET-4R (CB1) (b); C standard caffeic acid (a) and caffeic acid supplemented CB1 strain (b); D standard ferulic acid (a) and ferulic acid supplemented CB1 strain (b). Peak 1, cinnamic acid; peak 2, p-coumaric acid; peak 3, caffeic acid; peak 4, ferulic acid; peak 5, cinnamoyl-HSL; peak 6, p-coumaroyl-HSL; peak 7, caffeoyl-HSL; peak 8 , feruloyl-HSL 
fermentation without a precursor feeding process. We constructed the artificial de novo biosynthesis pathway for production of $p$-coumaroyl-HSL utilizing the rpaI gene addition with $p$-coumaroyl-CoA biosynthetic genes. The $p$-coumaroyl-CoA biosynthetic pathway genes are the tyrosine ammonia lyase gene $(\mathrm{tal})$ and $p$-coumaroylCoA ligase gene $(4 c l 2 n t)$, which converts tyrosine to $p$-coumaroyl-CoA through the $p$-coumaric acid. Tyrosine ammonia lyases (TAL, EC 4.3.1.25) identified from various sources can catalyze the direct formation of $p$-coumaric acid from tyrosine. We have already succeeded in synthesizing $p$-coumaric acid in $E$. coli from a simple medium without the addition of tyrosine using TAL from Saccharothrix espanaensis [24].

For the de novo synthesis of $p$-coumaroyl-HSL in $E$. coli, the only requirement was to replace the rpaI gene in the previously described plant polyketide expression vector (Additional file 1: Figure S3) [24-26]. This method is one of the advantages of assembling a biosynthetic pathway for a certain product; replacing a single enzyme gives a different product, the structure of which depends on its catalytic properties. The final pET-opT4R vector contains the tyrosine ammonia lyase, $p$-coumaroyl-CoA ligase, and $p$-coumaroyl-HSL synthase genes. The recombinant strain (DN1) that harbors the artificial biosynthetic gene cluster (pET-opT4R) was cultured in a modified synthetic medium (SM) [27]. The $p$-coumaroyl-HSL peak was detected in the culture broth of the DN1 strain by HPLC. Additionally, the DN1 strain was investigated using metabolite pattern analyses based on the culture times, until the production of $p$-coumaroyl-HSL was saturated after $15 \mathrm{~h}$. The amount of $p$-coumaroyl-HSL reached $19.4 \pm 2.0 \mathrm{mg} / \mathrm{L}(78.5 \mu \mathrm{M})$ at $15 \mathrm{~h}$ (Fig. 5). This productivity shows a remarkable improvement over the titers $(\sim 10 \mu \mathrm{M})$ of the original producer, a R. palustris [7].

\section{Improved production of $p$-coumaroyl-HSL in a tyrosine overproducing $E$. coli strain}

$p$-Coumaric acid is the pivotal intermediate of the plant phenylpropanoid pathway starting from the deamination of tyrosine. Thus tyrosine serves as an immediate endogenous precursor to the $p$-coumaroyl-HSL biosynthesis pathway. Recently, we reported engineered L-tyrosine overproducing $E$. coli $\triangle \mathrm{COS} 1$ strains via a deregulating of the aromatic amino acid biosynthesis pathway [27]. The tyrosine producer, E. coli $\triangle C O S 1$, was engineered on the genome to overexpress the feedback inhibition resistant (fbr) derivative genes of 3-deoxyD-arabinoheptulosonate-7-phosphate synthase (aro $G^{f b r}$ ) and chorismate mutase $\left(t y r A^{f b r}\right)$ in the repressor gene (tyrR) deletion strain. The tyrosine-overproducing strains showed a substantial capacity for $p$-coumaric acid, caffeic acid and ferulic acid biosynthesis [27]. Therefore, it

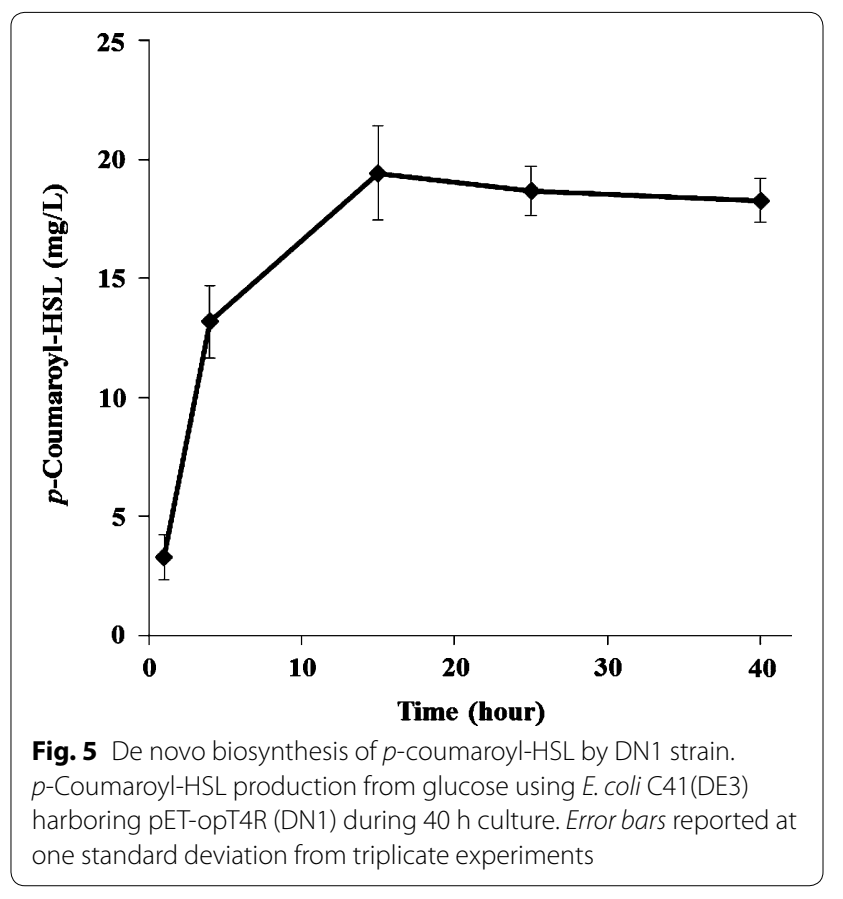

is a suitable platform strain for the production of other tyrosine-derived aromatic compounds, using the phenolic acids as precursors.

Using the same experimental conditions described above, the tyrosine-overproducing $E$. coli $\triangle \mathrm{COS} 1$ strain harboring the pET-opT4R vector (DN2) produced more than $60.9 \pm 0.5 \mathrm{mg} / \mathrm{L}$ of $p$-coumaroyl-HSL, an increase of $326 \%$ over the parental strain DN1 (Fig. 6). At the same time, an expected amount of accumulated $p$-coumaric acid was also identified (Additional file 1: Figure S4). The result means that extra $p$-coumaric acid is not well converted to $p$-coumaroyl-HSL, and accumulates in the cell. Therefore, the metabolic flow to $p$-coumaroyl-HSL may be interfered by a SAM shortage during the accumulation of $p$-coumaric acid in the tyrosine-overproducing cell. Most of the HSL uses a SAM as the HSL ring donor. SAM is produced from L-methionine and ATP catalyzed by methionine adenosyltransferase (MAT) in vivo. Previous studies reported that the SAM production is improved when supplemented with excessive L-methionine in a MAT overexpressing yeast strain [28]. In order to investigate the acceleration of the metabolic flux to $p$-coumaroyl-HSL via the SAM cycle, we supplied final $1 \mathrm{mM}$ of SAM and L-methionine to the culture medium of the DN1 and DN2 strains, respectively. Analysis of the product after $25 \mathrm{~h}$ showed that the production of the $p$-coumaroyl-HSL reached up to $93.4 \pm 0.6 \mathrm{mg} / \mathrm{L}$ from the $1 \mathrm{mM} S A M$ and $142.5 \pm 1.0 \mathrm{mg} / \mathrm{L}$ from the $1 \mathrm{mM}$ L-methionine in the DN2 strain, respectively (Fig. 6). 


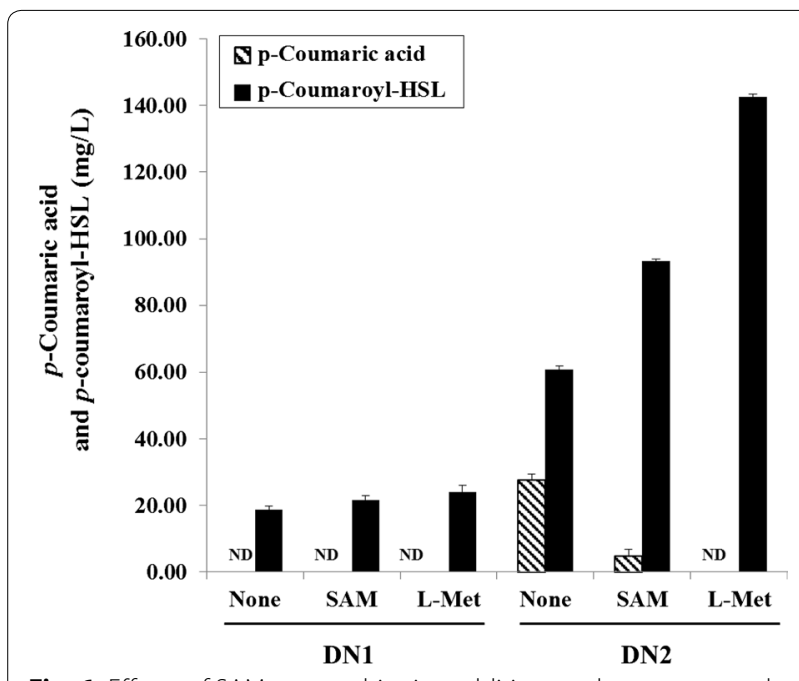

Fig. 6 Effects of SAM or L-methionine addition on the $p$-coumaroylHSL production. The data were obtained after $25 \mathrm{~h}$ fermentation with the addition of $1 \mathrm{mM} \mathrm{SAM}$ or $1 \mathrm{mM}$ L-methionine on the SM media of DN1 and DN2 strains, respectively. Error bars reported at one standard deviation from triplicate experiments. ND means not detected on the HPLC profile

The production levels of $p$-coumaroyl-HSL from the tyrosine overproducing DN2 strain represented improvements of 152 and $234 \%$ over the control culture, respectively. On the other hand, the fed SAM and L-methionine did not affect the production of $p$-coumaroyl-HSL in the DN1 strain (Fig. 6). The maximum production of $p$-coumaroyl-HSL from the DN1 strain was $21.7 \pm 1.2$ and $24.0 \pm 2.1 \mathrm{mg} / \mathrm{L}$, respectively, when $1 \mathrm{mM}$ SAM or L-methionine were fed. However, when tyrosine was fed together with SAM or L-methionine to the culture medium of the DN1 strain, the production of $p$-coumaroyl-HSL was $16.8 \pm 0.5$ and $122.0 \pm 1.0 \mathrm{mg} / \mathrm{L}$, respectively (Additional file 1: Figure S6). These results mean that the tyrosine and methionine are limiting factors to the production of $p$-coumaric acid and $p$-coumaroyl-HSL, respectively.

We found a remarkable improvement in $p$-coumaroyl-HSL synthesis when the tyrosine overproducing DN2 strain was grown in L-methionine containing media, and the DN1 strain was grown in the L-methionine and tyrosine containing media. These results indicate that the elevated L-methionine assimilation pathway allows for the metabolic flux improvement of extra $p$-coumaric acid, originating from tyrosine, converting it to $p$-coumaroyl-HSL. Therefore the best metabolic engineered strains for $p$-coumaroyl-HSL production would need to activate the SAM overproducing pathway, such as enhancement of the SAM synthase gene $(\operatorname{met} K)$ activity [28, 29]. The balanced metabolic flux of tyrosine and the SAM overproducing pathway could increase intracellular $p$-coumaroyl-HSL in the heterologous host.

\section{Conclusions}

The system in this study successfully demonstrated the de novo synthesis of a quorum sensing molecule, $p$-coumaroyl-HSL, using an artificial biosynthetic pathway in the heterologous host, E. coli. Further, the production of $p$-coumaroyl-HSL from a tyrosine overproducing strain (DN2) by feeding of SAM and L-methionine was determined to be $422 \%$ and $650 \%$ over the E. coli $\mathrm{DN} 1$, respectively. The titers of the $p$-coumaroyl-HSL reached up to $93.4 \pm 0.6 \mathrm{mg} / \mathrm{L}$ from $1 \mathrm{mM} \mathrm{SAM}$, and $142.5 \pm 1.0 \mathrm{mg} / \mathrm{L}$ from $1 \mathrm{mM}$ L-methionine, respectively, after $25 \mathrm{~h}$ of culturing in a glucose containing minimal medium. In addition, we demonstrated the bioconversion production of phenylacetyl-HSL analogs including cinnamoyl-HSL, $p$-coumaroyl-HSL, caffeoyl-HSL, and feruloyl-HSL in E. coli.

\section{Methods}

\section{Chemicals}

Cinnamic acid, $p$-coumaric acid, caffeic acid, ferulic acid, and $N$-( $p$-Coumaroyl)-L-homoserine lactone ( $p$-coumaroyl-HSL) were purchased from Sigma-Aldrich (USA) as substrates for feeding experiments and as standards for compound identification by HPLC. Also, adenosine triphosphate (ATP), Coenzyme A (CoA), L-methionine and $S$-adenosyl methionine (SAM) were purchased from Sigma-Aldrich for assay of enzymes activity.

\section{DNA manipulation}

The restriction enzymes (NEB; Takara), a nPfu-Forte DNA polymerase (Enzynomics, Korea), an ligation mix (Takara), were used according to the instructions provided by the manufacturers. The codon optimized tyrosine ammonia lyase gene (tal) from Saccharothrix espanaensis was synthesized by DNA 2.0, previously [30]. Codon optimization and synthesis of the $p$-coumaroyl CoA ligase $4 C L 2$ gene $(4 c l 2 n t)$ from Nicotiana tabacum (GenBank U50846.1) was performed with the GeneGPS $^{\mathrm{TM}}$ program (DNA2.0). Also, the HSL synthase gene rpaI from Rhodopseudomonas palustris (GenBank BX572593.1) were codon optimized and synthesized by Bioneer (Korea). The synthesized sequences are described in the supporting information.

\section{Expression and purification of 4CL2nt and Rpal proteins}

E. coli C41(DE3) [31] containing 4cl2nt and rpaI gene was grown overnight $\left(37^{\circ} \mathrm{C}\right)$ in $5 \mathrm{ml} \mathrm{LB}$ medium containing $50 \mu \mathrm{g} / \mathrm{ml}$ kanamycin, respectively. The broth of the cultures $(5 \mathrm{~mL})$ was used to inoculate $1 \mathrm{~L}$ flasks containing $300 \mathrm{~mL}$ of LB medium. The expressed proteins in the 
supernatant were purified by affinity chromatography using a Ni-NTA bead column (QIAGEN) according to the instructions provided by the manufacturers. After elution with $250 \mathrm{mM}$ imidazole buffer, the solutions containing the 4CL2nt and RpaI, respectively, were dialyzed against a Tris- $\mathrm{HCl}$ buffer $(50 \mathrm{mM}, \mathrm{pH} 7.4)$ containing $0.1 \mathrm{mM}$ EDTA, $0.1 \mathrm{mM}$ DTT and $10 \%$ glycerol. Typically, the enzymes purity were $>90 \%$, as determined by sodium dodecyl sulfate-polyacrylamide gel electrophoresis stained with Coomassie blue (Additional file 1: Figure S5).

\section{Assay of Rpal activity}

Reaction mixtures $(500 \mu \mathrm{L})$ containing Tris- $\mathrm{HCl}$ (50 mM, pH 7.4), $\mathrm{MgCl}_{2}$ (10 mM), ATP (1 mM), CoA $(0.2 \mathrm{mM})$, SAM $(0.1 \mathrm{mM})$ and each of phenolic acids (cinnamic acid, $p$-coumaric acid, caffeic acid and ferulic acid; $0.6 \mathrm{mM})$ with/without both 4CL2nt $(5 \mu \mathrm{M})$ and RpaI $(5 \mu \mathrm{M})$ were incubated at $30{ }^{\circ} \mathrm{C}$ for $1 \mathrm{~h}$. The reaction mixtures were extracted with an equal volume of ethyl acetate. The ethyl acetate was dried and resuspended in $100 \mu \mathrm{L}$ of methanol. The Twenty microliters of samples were applied to a J'sphere ODS-H80 column $(4.6 \times 150 \mathrm{~mm}$ i.d., $5 \mu \mathrm{m}$; YMC, Japan $)$ using a highperformance liquid chromatography (HPLC) system $\left[\mathrm{CH}_{3} \mathrm{CN}-\mathrm{H}_{2} \mathrm{O}\right.$ (0.05\% trifluoroacetic acid), $10-100 \%$ acetonitrile $\left(\mathrm{CH}_{3} \mathrm{CN}\right)$ for $25 \mathrm{~min}$ at flow rate of $1 \mathrm{~mL} /$ min; Dionex, USA] equipped with a photodiode array detector. A liquid chromatography -mass spectrometry (LC-MS) was performed using an LTQ XL linear ion trap (Thermo Scientific, USA) equipped with an electrospray ionization (ESI) source that was coupled to a rapid separation LC (RSLC; ultimate 3000, Thermo Scientific) system (ESI-LC-MS) using a HSS T3 column (Waters, UK) $(2.1 \times 150 \mathrm{~mm} ; 2.5 \mu \mathrm{m}$ particle size $)$ with a linear gradient of the binary solvent system under the same HPLC conditions as described above. The data-dependent mass spectrometry experiments were controlled using the menu driven software provide with the Xcalibur system (version 2.2 SP1.48; Thermo Scientific). The compounds were identified through comparisons with the standard compounds using the observed retention time, ultraviolet spectra, and mass chromatogram. The conversion rate was calculated based on a quantitative comparison with the peak areas of absorbance at $300 \mathrm{~nm}$ of remained substrates and the products after enzyme reactions.

\section{Construction of bioconversion vectors ( $\mathrm{pET}-4 \mathrm{R})$ and de novo synthesis vector ( $\mathrm{pET}$-opT4R)}

In order to construct an expression vector containing the $4 c l 2 n t$ and rpaI genes, the two genes were independently cloned into the NdeI and XhoI sites on pET-28a(+), which resulted in pET-his4CL2nt and pET-opRpaI, respectively. In order to assemble the pET-4R vector, the $4 c l 2 n t$ coding regions was amplified using the pET-4CL2nt as a template with the primers Npac (the sequence is located upstream of the T7 promoter region of the pET vector and contains the designed PacI site: TTAATTAATCGC CGCGACAATTTGCGACGG) and Cspe (the sequence is located downstream of the T7 terminator region of the $\mathrm{pET}$ vector and contains the designed SpeI site: ACT AGTTCCTCCTTTCAGCAAAAAACCCCTC). The rpaI coding regions was amplified using the pET-opRpaI as a template with the Nspe (the sequence is located upstream of the T7 promoter region of the pET vector and contains the designed SpeI site: ACTAGTAGGTTGAG GCCGTTGAGCACCGCC) and Cpac (the sequence is located downstream of the T7 terminator region of the pET vector and contains the designed PacI site: TTAAT TAATGCGCCGCTACAGGGCGCGTCC) primers. Two amplified fragments containing the $4 c l 2 n t$ and rpaI coding regions, respectively, were digested with corresponding sites and cloned into pET-28a(+) using NdeI, SpeI and $\mathrm{XhoI}$ sites, which resulted in pET-4R (Table 1; Additional file 1: Figure S3). In order to construct an expression vector containing the tal, $4 c l 2 n t$ and rpaI genes, the tal gene were also cloned into the NdeI and XhoI sites on pET-28a $(+)$, which resulted in pET-opTAL [12]. In order to assemble the pET-opT4R vector, the tal coding region was amplified using the pET-opTAL as a template with the primers opTAL-F (5'-CATATGACCCAGGTG GTTGAACGCC-3') and Cpac. As constructed above, three amplified fragments containing the tal, $4 c l 2 n t$ and rpaI coding regions, respectively, were digested with corresponding sites and cloned into pET-28a(+) using NdeI, PacI, SpeI and XhoI sites, which resulted in pET-opT4R (Table 1; Additional file 1: Figure S3). The gene sequences and orientations were verified via sequencing after each round of cloning, and the recombinant plasmids were transformed into $E$. coli for gene expression.

\section{Production of phenylacetyl-HSL analogs by $E$. coli}

Recombinant E. coli strain (CB1) with the rpaI and 4cl2nt genes (pET-4R) $37{ }^{\circ} \mathrm{C}$ in a Luria-Bertani (LB) medium containing $50 \mu \mathrm{g} / \mathrm{mL}$ kanamycin. The overnight culture was inoculated (1.5\%) into a fresh LB medium supplemented with the same concentration of kanamycin. The culture was grown at $37{ }^{\circ} \mathrm{C}$ to an optical density of $600 \mathrm{~nm}$ (OD 600) of 0.6. Then, IPTG was added to the final concentration of $1 \mathrm{mM}$, and the culture was incubated for $6 \mathrm{~h}$. The cells were harvested by centrifugation, suspended, and incubated at $26{ }^{\circ} \mathrm{C}$ until $36 \mathrm{~h}$ in a modified synthetic medium (SM; $3 \mathrm{~g} / \mathrm{L} \mathrm{KH}_{2} \mathrm{PO}_{4}, 7.3 \mathrm{~g} / \mathrm{L}$ $\mathrm{K}_{2} \mathrm{HPO}_{4}, 8.4 \mathrm{~g} / \mathrm{L}$ MOPS, $2 \mathrm{~g} / \mathrm{L} \mathrm{NH} \mathrm{N}_{4} \mathrm{Cl}, 0.5 \mathrm{~g} / \mathrm{L} \mathrm{NaCl}$, 
$0.1 \mathrm{ml} / \mathrm{L}$ Trace elements, $5 \mathrm{~g} / \mathrm{L}\left(\mathrm{NH}_{4}\right)_{2} \mathrm{SO}_{4}, 5 \mathrm{~g} / \mathrm{L} \mathrm{MgSO}_{4}$, and supplemented with $15 \mathrm{~g} / \mathrm{L}$ glucose, $1 \mathrm{mM}$ IPTG and $50 \mu \mathrm{g} / \mathrm{mL}$ kanamycin) [27, 32]. For the bioconversion experiments, the cultures were supplemented with cinnamic acid, $p$-coumaric acid, caffeic acid and ferulic acid (final concentration: $30 \mathrm{mg} / \mathrm{L}$ ), respectively. The samples were collected after $24 \mathrm{~h}$ and analyzed by HPLC. For the detection of cinnamoyl-HSL, $p$-coumaroyl-HSL, caffeoyl-HSL and feruloyl-HSL, $1 \mathrm{~mL}$ of cell-free culture supernatants were filtered through $0.2 \mu \mathrm{m}$ cellulose membrane syringe filters (Sartorius) and twenty microliters of samples were applied to a SunFire ${ }^{\mathrm{TM}} \mathrm{C} 18$ column $(250 \times 4.6 \mathrm{~mm}, 5 \mu \mathrm{m}$; Waters, USA $)$ using a HPLC system $\left[\mathrm{CH}_{3} \mathrm{CN}-\mathrm{H}_{2} \mathrm{O}(0.05 \%\right.$ trifluoroacetic acid), $10-60 \%$ acetonitrile $\left(\mathrm{CH}_{3} \mathrm{CN}\right)$ for $25 \mathrm{~min}$ at flow rate of $1 \mathrm{~mL} / \mathrm{min}$; Dionex, USA] equipped with a photodiode array detector. Further, the recombinant E. coli strains (DN1) and tyrosine overproducing strain (DN2) that harbored the pET-opT4R plasmid were cultured via the same method as described bioconversion. For the SAM, L-methionine and tyrosine feeding experiments, the compounds were added at concentration of $1 \mathrm{mM}$, respectively, to the fermentative media after the IPTG induction period. The detection and quantification of $p$-coumaroyl-HSL in $E$. coli was carried out as above described.

\section{Purification and structural elucidation of the phenylacetyl-HSL analogs}

Further, the recombinant E. coli strains that harbored the pET-4R plasmid (DN1 and DN2) were cultured via the same method as described earlier with caffeic acid or ferulic acid, the culture volume and time were increased to $2 \mathrm{~L}$ for $60 \mathrm{~h}$. When we supplemented with caffeic acid $(10 \mathrm{mg} / \mathrm{L})$ and ferulic acid $(15 \mathrm{mg} / \mathrm{L})$, respectively, the EtOAc-soluble material was further purified by reversephase HPLC (Waters Co., USA) using the YMC J'sphere ODS-H80 $(10 \times 250 \mathrm{~mm}, 3 \mathrm{~mL} / \mathrm{min})$ with a linear gradient from 20 to $100 \% \mathrm{CH}_{3} \mathrm{CN}$ containing $0.05 \%$ TFA in order to yield caffeoyl-HSL $(1.2 \mathrm{mg})$ and feruloyl-HSL (3.6 mg). The E. coli strain that harbored the pET-opT4R plasmid was cultured via the same method as described earlier, but the culture volume and time were increased to $2.4 \mathrm{~L}$ until $60 \mathrm{~h}$. The $p$-coumaroyl-HSL was purified $22.8 \mathrm{mg}$. The structural elucidation of the purified compounds was undertaken using ${ }^{1} \mathrm{H}$ and ${ }^{13} \mathrm{C}$ NMR spectroscopy. The NMR experiments were performed on a Bruker AVANCE spectrometer (700, $900 \mathrm{MHz}$; Bruker Inc., USA). The structure of $p$-coumaroyl-HSL was determined based on the ${ }^{1} \mathrm{H}$ NMR data with the values reported in the literature [7]; also caffeoyl-HSL and feruloyl-HSL were determined based on the 1D, 2D NMR data (Additional file 1: Table S1).

\section{Additional file}

Additional file 1. Further details of relevance to this study.

\section{Authors' contributions}

SK and $J L$ performed the experiments and wrote the manuscript. JL and JJ coperformed the experiments on the metabolite analysis. $\mathrm{BH}$ and $\mathrm{JJ}$ contributed general advice, particularly on the metabolite analysis and resource support. YH designed all the experiments and wrote the manuscript. All authors read and approved the final manuscript.

\section{Author details}

${ }^{1}$ Chemical Biology Research Center, Korea Research Institute of Bioscience and Biotechnology, 30 Yeongudanji-ro, Ochang-eup, Chungbuk 363-883, Republic of Korea. ${ }^{2}$ Department of Pharmacy Graduate School, Chungbuk National University, Cheongju 361-763, Republic of Korea.

\section{Acknowledgements}

This work was supported in part by Global R\&D Center program (NRF2010-00719), NRF and by the Next-Generation BioGreen 21 Program (SSAC, PJ011084012015), RDA Republic of Korea. We thank the Gyeonggi Bio Center for the HRESIMS data and the KBSI, Ochang, for the NMR data.

\section{Competing interests}

The authors declare that they have no competing interests.

Received: 28 July 2015 Accepted: 6 November 2015

Published online: 25 November 2015

\section{References}

1. Miller MB, Bassler BL. Quorum sensing in bacteria. Annu Rev Microbiol. 2001;55:165-99.

2. Waters CM, Bassler BL. Quorum sensing: cell-to-cell communication in bacteria. Annu Rev Cell Dev Biol. 2005;21:319-46.

3. Hastings JW, Greenberg EP. Quorum sensing: the explanation of a curious phenomenon reveals a common characteristic of bacteria. J Bacteriol. 1999;181:2667-8.

4. Surette MG, Miller MB, Bassler BL. Quorum sensing in Escherichia coli, Salmonella typhimurium, and Vibrio harveyi: a new family of genes responsible for autoinducer production. Proc Natl Acad Sci USA. 1999;96:1639-44.

5. Schaefer AL, Val DL, Hanzelka BL, Cronan JE Jr, Greenberg EP. Generation of cell-to-cell signals in quorum sensing: acyl homoserine lactone synthase activity of a purified Vibrio fischeri Luxl protein. Proc Natl Acad Sci USA. 1996;93:9505-9.

6. Rutherford ST, Bassler BL. Bacterial quorum sensing: its role in virulence and possibilities for its control. Cold Spring Harb Perspect Med. 2012;2(11):a012427.

7. Schaefer AL, Greenberg EP, Oliver CM, Oda Y, Huang JJ, Bittan-Banin G, Peres CM, Schmidt S, Juhaszova K, Sufrin JR, Harwood CS. A new class of homoserine lactone quorum-sensing signals. Nature. 2008;454:595-9.

8. Hirakawa H, Oda Y, Phattarasukol S, Armour CD, Castle JC, Raymond CK, Lappala CR, Schaefer AL, Harwood CS, Greenberg EP. Activity of the Rhodopseudomonas palustris p-coumaroyl-homoserine lactone-responsive transcription factor RpaR. J Bacteriol. 2011;193:2598-607.

9. Pan C, Oda Y, Lankford PK, Zhang B, Samatova NF, Pelletier DA, Harwood CS, Hettich RL. Characterization of anaerobic catabolism of $p$-coumarate in Rhodopseudomonas palustris by integrating transcriptomics and quantitative proteomics. Mol Cell Proteomics. 2008;7:938-48.

10. Ahlgren NA, Harwood CS, Schaefer AL, Giraud E, Greenberg EP. Arylhomoserine lactone quorum sensing in stem-nodulating photosynthetic bradyrhizobia. Proc Natl Acad Sci USA. 2011;108:7183-8.

11. More MI, Finger LD, Stryker JL, Fuqua C, Eberhard A, Winans SC. Enzymatic synthesis of a quorum-sensing autoinducer through use of defined substrates. Science. 1996;272:1655-8. 
12. Tang K, Zhang XH. Quorum quenching agents: resources for antivirulence therapy. Mar Drugs. 2014;12:3245-82.

13. Seyedsayamdost MR, Case RJ, Kolter R, Clardy J. The Jekyll-and-Hyde chemistry of Phaeobacter gallaeciensis. Nat Chem. 2011;3:331-5.

14. Rasmussen TB, Bjarnsholt $T$, Skindersoe ME, Hentzer M, Kristoffersen $P$, Kote M, Nielsen J, Eberl L, Givskov M. Screening for quorum-sensing inhibitors (QSI) by use of a novel genetic system, the QSI selector. J Bacteriol. 2005;187:1799-814.

15. Geske GD, Wezeman RJ, Siegel AP, Blackwell HE. Small molecule inhibitors of bacterial quorum sensing and biofilm formation. J Am Chem Soc. 2005;127:12762-3.

16. Geske GD, O’Neill JC, Miller DM, Wezeman RJ, Mattmann ME, Lin Q, Blackwell HE. Comparative analyses of $\mathrm{N}$-acylated homoserine lactones reveal unique structural features that dictate their ability to activate or inhibit quorum sensing. ChemBioChem. 2008;9:389-400.

17. Geske GD, O'Neill JC, Miller DM, Mattmann ME, Blackwell HE. Modulation of bacterial quorum sensing with synthetic ligands: systematic evaluation of N-acylated homoserine lactones in multiple species and new insights into their mechanisms of action. J Am Chem Soc. 2007;129:13613-25.

18. Gamby S, Roy V, Guo M, Smith JA, Wang J, Stewart JE, Wang X, Bentley WE, Sintim HO. Altering the communication networks of multispecies microbial systems using a diverse toolbox of Al-2 analogues. ACS Chem Biol. 2012;7:1023-30.

19. Koh CL, Sam CK, Yin WF, Tan LY, Krishnan T, Chong YM, Chan K-G. Plantderived natural products as sources of anti-quorum sensing compounds. Sensors. 2013;13:12

20. Galloway WR, Hodgkinson JT, Bowden SD, Welch M, Spring DR. Quorum sensing in Gram-negative bacteria: small-molecule modulation of AHL and Al-2 quorum sensing pathways. Chem Rev. 2011;111:28-67.

21. Palmer AG, Blackwell HE. Deciphering a protolanguage for bacteria-host communication. Nat Chem Biol. 2008;4:452-4.

22. Parsek MR, Val DL, Hanzelka BL, Cronan JE Jr, Greenberg EP. Acyl homoserine-lactone quorum-sensing signal generation. Proc Natl Acad Sci USA. 1999;96:4360-5
23. Lee D, Douglas CJ. Two divergent members of a tobacco 4-coumarate:coenzyme A ligase (4CL) gene family. cDNA structure, gene inheritance and expression, and properties of recombinant proteins. Plant Physiol. 1996;112:193-205.

24. Kang SY, Lee JK, Choi O, Kim CY, Jang JH, Hwang BY, Hong YS. Biosynthesis of methylated resveratrol analogs through the construction of an artificial biosynthetic pathway in E. coli. BMC Biotechnol. 2014;14:67.

25. Choi O, Wu CZ, Kang SY, Ahn JS, Uhm TB, Hong YS. Biosynthesis of plantspecific phenylpropanoids by construction of an artificial biosynthetic pathway in Escherichia coli. J Ind Microbiol Biotechnol. 2011;38:1657-65.

26. Choi O, Lee JK, Kang SY, Pandey RP, Sohng JK, Ahn JS, Hong YS. Construction of artificial biosynthetic pathways for resveratrol glucoside derivatives. J Microbiol Biotechnol. 2014;24:614-8.

27. Kang SY, Choi O, Lee JK, Ahn JO, Ahn JS, Hwang BY, Hong YS. Artificial de novo biosynthesis of hydroxystyrene derivatives in a tyrosine overproducing Escherichia coli strain. Microb Cell Fact. 2015;14:78.

28. Hu H, Qian J, Chu J, Wang Y, Zhuang Y, Zhang S. Optimization of L-methionine feeding strategy for improving S-adenosyl-L-methionine production by methionine adenosyltransferase overexpressed Pichia pastoris. Appl Microbiol Biotechnol. 2009;83:1105-14.

29. Chu J, Qian J, Zhuang Y, Zhang S, Li Y. Progress in the research of S-adenosyl-L-methionine production. Appl Microbiol Biotechnol. 2013;97:41-9.

30. Kang SY, Choi O, Lee JK, Hwang BY, Uhm TB, Hong YS. Artificial biosynthesis of phenylpropanoic acids in a tyrosine overproducing Escherichia coli strain. Microb Cell Fact. 2012;11:153.

31. Miroux B, Walker JE. Over-production of proteins in Escherichia coli: mutant hosts that allow synthesis of some membrane proteins and globular proteins at high levels. J Mol Biol. 1996;260:289-98.

32. Zhang H, Stephanopoulos G. Engineering E. coli for caffeic acid biosynthesis from renewable sugars. Appl Microbiol Biotechnol. 2013;97:3333-41.

\section{Submit your next manuscript to BioMed Central and take full advantage of:}

- Convenient online submission

- Thorough peer review

- No space constraints or color figure charges

- Immediate publication on acceptance

- Inclusion in PubMed, CAS, Scopus and Google Scholar

- Research which is freely available for redistribution

Submit your manuscript at

www.biomedcentral.com/submit

C Biomed Central 